\title{
Paclitaxel induces apoptosis of esophageal squamous cell carcinoma cells by downregulating STAT3 phosphorylation at Ser727
}

\author{
XIAOLONG ZHANG ${ }^{1,2^{*}}$, XIAOYI WU ${ }^{2 *}$, FANGLING ZHANG ${ }^{3 *}$, SHOUYONG MO ${ }^{2,4}$, YUANYUAN LU ${ }^{2}$, \\ WEI WEI ${ }^{2}$, XIAOLING CHEN ${ }^{2}$, LINHUA LAN ${ }^{2}$, BIN LU $^{2}$ and YONGZHANG LIU ${ }^{2}$ \\ ${ }^{1}$ Department of Anesthesia and Critical Care, The Second Affiliated Hospital and Yuying Children's Hospital of \\ Wenzhou Medical University, Wenzhou, Zhejiang 325027; ${ }^{2}$ Protein Quality Control and Diseases Laboratory, Cancer Center, \\ School of Laboratory Medicine and Life Sciences, Wenzhou Medical University; ${ }^{3}$ The Second Clinical Medical School, \\ Wenzhou Medical University, Wenzhou, Zhejiang 325035; ${ }^{4}$ The Fifth Affiliated Hospital of \\ Wenzhou Medical University, Lishui, Zhejiang 323000, P.R. China
}

Received September 26, 2016; Accepted March 7, 2017

DOI: $10.3892 /$ or.2017.5503

\begin{abstract}
Paclitaxel induces apoptosis in a variety of cancer cells. However, the mechanism of paclitaxel inducing apoptosis in human esophageal squamous cell carcinoma (ESCC) remains to be defined. In this study, we found that paclitaxelinduced apoptosis by increasing the relevant apoptosis protein expression and the release of cytochrome $c$ via downregulation of signal transducer and activator of transcription 3 (STAT3) and phospho-STAT3 (Ser727). In addition, paclitaxel treatment of ESCC cells EC-1 and Eca-109 led to marked mitochondrial membrane potential depolarization and significantly increasing of reactive oxygen species. Moreover, paclitaxel treatment resulted in the inhibition of mitochondrial respiration. In conclusion, our findings reveal that paclitaxel induced apoptosis in both EC-1 and Eca-109 cells through the reduction of STAT3 and phospho-STAT3 (Ser727) level, and suggest that paclitaxel may be of therapeutic potential in the treatment of ESCC through the induction of mitochondrial apoptosis in ESCC cells.
\end{abstract}

\section{Introduction}

Esophageal cancer is one of the most common and aggressive types of cancer worldwide, it is ranked as the 8th

Correspondence to: Professor Bin Lu or Professor Yongzhang Liu, Protein Quality Control and Diseases Laboratory, Cancer Center, School of Laboratory Medicine and Life Sciences, Wenzhou Medical University, Wenzhou, Zhejiang 325035, P.R. China

E-mail: lubmito@wmu.edu.cn

E-mail: lyz@wmu.edu.cn

"Contributed equally

Key words: paclitaxel, esophageal squamous cell carcinoma, STAT3, apoptosis, mitochondria in morbidity and 6th in cancer-related mortality $(1,2)$. Esophageal squamous cell carcinoma (ESCC) and esophageal adenocarcinoma (EA) are the two major histological types of esophageal cancer (3). However, ESCC is the main subtype of esophageal cancer and comprises $\sim 70 \%$ of cases worldwide $(4,5)$. The incidence of ESCC has been increasing steadily in high-incidence areas such as northern China, Iran and South Africa (4-6), and the overall survival still remains extremely poor due to lack of effective biomarkers for early diagnosis, and therapeutic target. Therefore, a better understanding of the mechanism of this malignancy to identify and characterize novel therapies is definitely necessary and of great urgency.

Paclitaxel is a cytotoxic apoptosis inducer and antineoplastic drug against a broad-spectrum of cancers such as ovarian cancer, lung cancer, breast cancer and melanoma (7). As a single agent, paclitaxel has been shown to have a response rate of $32 \%$ in esophageal cancer (8). In addition, several phase II studies indicated that paclitaxel-based regimens play an important role in patients with locally advanced and metastatic esophageal cancer (9-11). Moreover, paclitaxel may increase the sensitivity of tumor cells to radiation (12-14). Signal transducers and activators of transcription 3 (STAT3) involved in cell proliferation, differentiation, cell survival and death in response to various signaling through the phosphorylation and nuclear translocation $(15,16)$. STAT3 was constitutively activated in cancer tissues of ESCC (17). Wegrzyn et al found that STAT3 may translocate to mitochondria, and the mitochondrial STAT3 (mtSTAT3) is important for the functions of mitochondrial electron transport chain (ETC) since the activities of complex I and II of mitochondrial ETC were significantly decreased in $\mathrm{STAT}^{-/}$cells (18). Moreover, mtSTAT3 supports Ras-dependent malignant transformation by regulating the glycolysis and oxidative phosphorylation (OXPHOS) of cancer cells (19). Previous studies on paclitaxel-induced apoptosis mainly focused on the mitochondrial apoptotic pathways (20-22). However, the regulatory mechanism of 
how it induces apoptosis and the role of STAT3 in ESCC cells remains to be further investigated.

\section{Materials and methods}

Reagents and antibodies. Paclitaxel was obtained from Sangon Biotech Co. (Shanghai, China). Horseradish peroxidase (HRP)-conjugated anti-rabbit, anti-mouse immunoglobulin $\mathrm{G}$, MTT Cell Proliferation and Cytotoxicity Assay kit, ROS Assay kit (DCFH-DA) and mitochondrial membrane potential (MMP) Assay kit (JC-1) were all obtained from Beyotime Biotech (Jiangshu, China). Annexin V-Fluorescein Isothiocyanate (FITC) Apoptosis Detection kit was obtained from BD Biosciences (Franklin Lakes, NJ, USA). BCA Protein Assay kit and Pierce ECL Western Blotting Substrate were both obtained from Thermo Fisher Scientific, Inc. (Waltham, MA, USA). Monoclonal antibody against $\beta$-actin was from Abmart (Shanghai, China). Polyclonal antibody against PARP, caspase-3, cleaved caspase-3, caspase-7, cleaved caspase-7, caspase-9, cleaved caspase-9, STAT3, phospho-STAT3 (Ser727) were all obtained from Cell Signaling Technology, Inc. (Beverly, MA, USA). Polyclonal antibody against cytochrome $c$, COX IV, GAPDH, VDAC were all purchased from Abcam (Cambridge, UK).

Cell lines and cell culture. The ESCC cell lines, EC-1 and Eca-109 were both purchased from the Cell Bank of the Chinese Academy of Sciences (Shanghai, China). Cells were grown in RPMI-1640 medium supplemented with $10 \%$ fetal bovine serum (FBS) (both from Invitrogen, Carlsbad, CA, USA) and antibiotics (100 U/ml penicillin and $100 \mu \mathrm{g} / \mathrm{ml}$ streptomycin) at $37^{\circ} \mathrm{C}$ in a humidified atmosphere of $5 \% \mathrm{CO}_{2}$.

Isolation of cytoplasmic and mitochondrial fractions. EC-1 and Eca-109 cells were treated with 12 and $20 \mathrm{nM}$ paclitaxel for $24 \mathrm{~h}$, respectively. The cells harvested were lysed in $2 \mathrm{ml} \mathrm{0.1X}$ isolation buffer (IB, $3.5 \mathrm{mM}$ Tris- $\mathrm{HCl}, 2.5 \mathrm{mM}$ $\mathrm{NaCl}, 0.5 \mathrm{mM} \mathrm{MgCl}_{2}$ and protease inhibitors, $\mathrm{pH}$ 7.8) and incubated on ice for $2 \mathrm{~min}$. The mixture was then transferred into the Dounce glass homogenizer. After more than $90 \%$ of cells were confirmed to be broken by using phase contrast microscope, $200 \mu 1$ 10X IB and 0.1X IB were added into the mixture for further lysing the cells. The lysate were then centrifuged at $1,000 \mathrm{x} \mathrm{g}$ for $3 \mathrm{~min}$, and the supernatant were transferred to a new Eppendorf tube, centrifuged at 15,000 x g for $2 \mathrm{~min}$, and the supernatant was cytoplasmic fraction and the pellet obtained was mitochondrial fraction which was resuspended in $200 \mu \mathrm{l}$ buffer A (10 mM Tris- $\mathrm{HCl}, \mathrm{pH} 7.9,1 \mathrm{mM}$ EDTA, $0.32 \mathrm{M}$ sucrose) and $2 \mu 1100 \mathrm{mM}$ PMSF.

3-(4,5-Dimethylthiazol-2-yl)-2,5-diphenyltetrazolium bromide (MTT) assay. For MTT assay, EC-1 and Eca-109 cells were seeded into 96 -well plates at a density of $2 \times 10^{3}$ cells/well and incubated at $37^{\circ} \mathrm{C}$ in a humidified atmosphere of $5 \% \mathrm{CO}_{2}$ overnight. Next day, EC-1 and Eca-109 cells were treated with 12 and $20 \mathrm{nM}$ paclitaxel for $0,24,48$ and $72 \mathrm{~h}$, respectively. Cells were stained with MTT reagent $(5 \mathrm{mg} / \mathrm{ml})$ for $4 \mathrm{~h}$, and the crystals produced were dissolved with DMSO. Once the crystals were dissolved completely, the plate was measured at $570 \mathrm{~nm}$ by the plate reader (Thermo Fisher Scientific, Inc.).
Apoptosis analysis. EC-1 and Eca-109 cells were seeded in a 6 -well plate at a density of $2 \times 10^{5}$ cells/well and treated with 12 and $20 \mathrm{nM}$ paclitaxel, respectively. Following treatment for $24 \mathrm{~h}$, the cells were collected, washed twice with ice-cold phosphate-buffered saline PBS and subsequently stained with Annexin V-FITC/PI for $20 \mathrm{~min}$ by incubation in the dark at room temperature. The samples were then analyzed immediately using a BD Accuri C6 flow cytometer (BD Biosciences).

Reactive oxygen species (ROS) determination. DCFH-DA detection kit was used to measure the intracellular ROS levels according to the manufacturer's instructions. Briefly, EC-1 and Eca-109 cells were treated with paclitaxel at concentrations of 12 and $20 \mathrm{nM}$, respectively. Following the treatment for $24 \mathrm{~h}$, cells were collected, washed with RPMI-1640 and incubated with $10 \mathrm{mM}$ DCFH-DA at $37^{\circ} \mathrm{C}$ for $15 \mathrm{~min}$ in the dark. The cells were then washed with RPMI-1640 and resuspended in $200 \mu \mathrm{l}$ RPMI-1640. The generation of intracellular ROS was analyzed by flow cytometry within $30 \mathrm{~min}$.

JC-1 fluorescence analysis. The MMP was measured by means of JC-1 staining as previously described (24). Briefly, EC-1 and Eca-109 cells treated with paclitaxel were incubated with $2.0 \mu \mathrm{M} \mathrm{JC}-1$ probe for $20 \mathrm{~min}$ at $37^{\circ} \mathrm{C}$ in the dark. The excess JC-1 probe was removed by washing cells with warm PBS and pelleted by centrifugation. Cell pellets were resuspended in $500 \mu \mathrm{l}$ PBS and the MMP was assayed by flow cytometry.

RNA interference. EC-1 and Eca-109 cells were transfected with control or STAT3 siRNA (Genepharma, Shanghai, China) using Lipofectamine 2000 (Life Technologies, Grand Island, NY, USA) in serum-free medium following manufacturer's instructions. Thirty-six hours post-transfection, cells were seeded on two 6-cm dishes, respectively. Then, cells were treated with or without indicated concentration of PTX (20 nM for EC-1 cells and $12 \mathrm{nM}$ for Eca-109 cells, respectively) for another $24 \mathrm{~h}$. Cells were collected and further subjected to western blot analysis with indicated antibodies. The following sequences for STAT3 siRNA are: STAT3-homo-398 sense, 5'-CCACUUUGGUGUUUCAUAATT-3' and antisense, 5'-UUAUGAAACACCAAAGUGGTT-3'; STAT3-homo-978 sense, 5'-GCAACAGAUUGCCUGCAUUTT-3' and antisense, 5'-AAUGCAGGCAAUCUGUUGCTT-3'; and control siRNA sense, 5'-UUCUCCGAACGUGUCACGUTT-3' and antisense, 5'-AGGUGACACGUUCGGAGAATT-3'.

Western blot analysis. EC-1 and Eca-109 cells were treated with or without paclitaxel for $24 \mathrm{~h}$, then trypsinized and collected in $1.5 \mathrm{ml}$ Eppendorf tubes, lysed in RIPA buffer (50 mM Tris-HCl, pH 7.4, 1\% Triton X-100, 1\% sodium deoxycholate, $0.1 \%$ SDS, $150 \mathrm{mM} \mathrm{NaCl}$ ) supplemented with protease inhibitor cocktail tablet, $\mathrm{NaF}(1 \mathrm{mM})$ and $\mathrm{Na}_{3} \mathrm{VO}_{4}(1 \mathrm{mM})$ for $15 \mathrm{~min}$ on ice and centrifuged at $18,000 \mathrm{x} \mathrm{g}$ for $20 \mathrm{~min}$ at $4^{\circ} \mathrm{C}$. Equal amount of protein from total lysates were resolved by $15 \%$ SDS-PAGE, the separated proteins were transferred onto nitrocellulose membrane (Bio-Rad Laboratories, Inc., Hercules, CA, USA) in Tris-glycine buffer. Blots were blocked at room temperature for $1.5 \mathrm{~h}$ in blocking buffer (5\% non-fat milk in TBST) on a shaker 
A
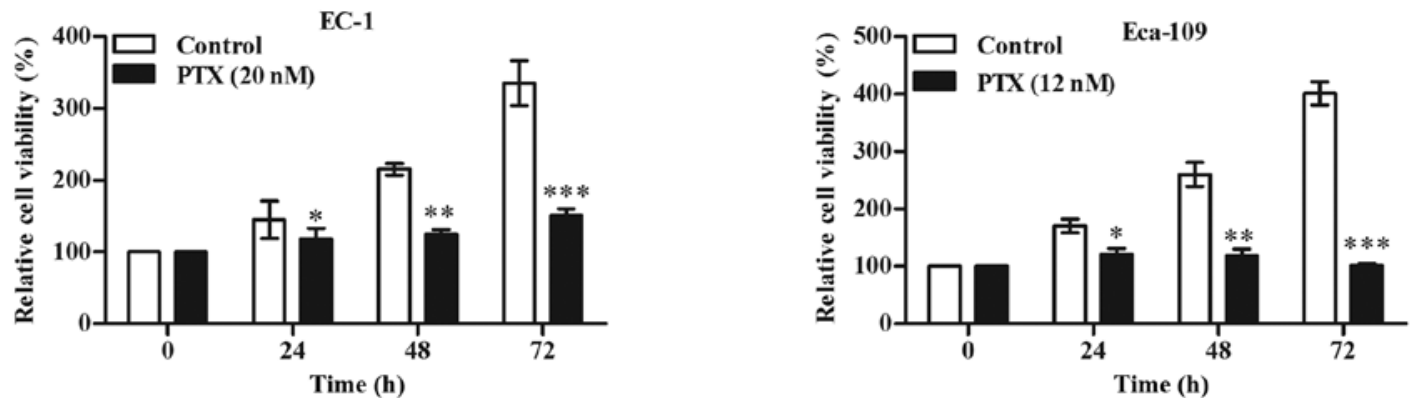

B EC-1
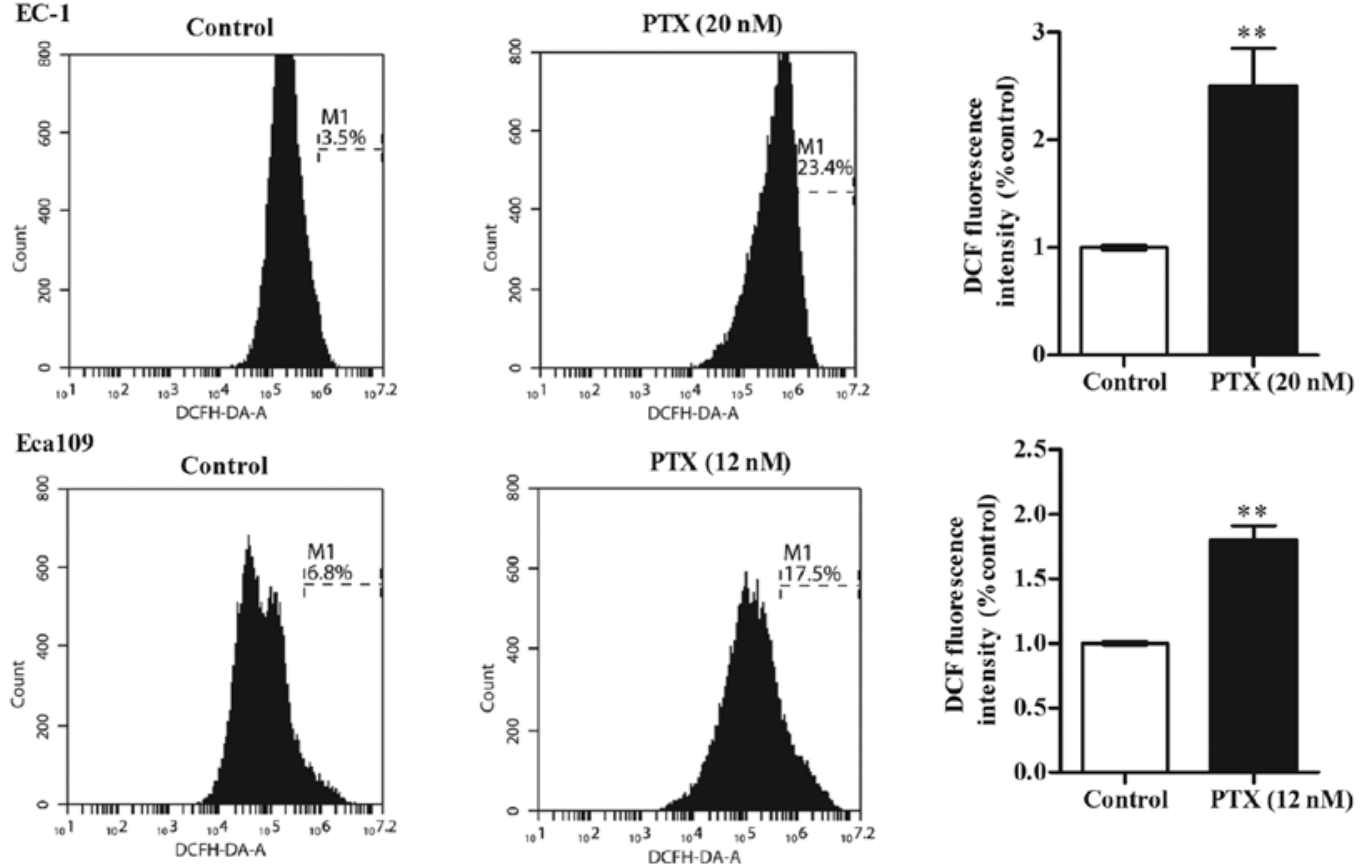

Figure 1. Paclitaxel inhibits EC-1 and Eca-109 cell growth and increases intracellular ROS in a time-dependent manner. (A) EC-1 and Eca-109 cells were treated with 20 and $12 \mathrm{nM}$ paclitaxel for $0,24,48$ and $72 \mathrm{~h}$, respectively. The cell viability was examined by performing an MTT assay. (B) The intracellular ROS production of EC-1 and Eca-109 cells induced by paclitaxel were analyzed by flow cytometry. ROS, reactive oxygen species. ${ }^{*}$ p $<0.05 ;^{* *}$ p $<0.01 ;{ }^{* * *}$ p $<0.001$.

and then incubated overnight at $4{ }^{\circ} \mathrm{C}$ with primary antibodies as indicated in the figures.After washing, membranes were incubated for $1 \mathrm{~h}$ with an anti-mouse or anti-rabbit Ig-HRP and detected with the ECL system. The optical density was quantified by the ImageJ software (National Institutes of Health, Bethesda, MA, USA).

OXPHOS assay. The Seahorse XF96 Extracellular Flux Analyzer (Seahorse Bioscience, North Billerica, MA, USA) was used to assay mitochondrial bioenergetics by measuring the oxygen consumption rate (OCR). The optimum number of cells was determined to be $20,000 /$ well and incubated in a $37^{\circ} \mathrm{C}$ $5 \% \mathrm{CO}_{2}$ incubator, and the calibrator plate was equilibrated in a non- $\mathrm{CO}_{2}$ incubator overnight. Next day, the medium was changed to Dulbecco's modified Eagle's medium (unbuffered DMEM, $25 \mathrm{mM}$ glucose, $1 \mathrm{mM}$ glutamine, $1 \mathrm{mM}$ sodium pyruvate) and equilibrated in a $37^{\circ} \mathrm{C} \mathrm{CO}_{2}$-free prep station for $40 \mathrm{~min}$. Then, oligomycin ( $1 \mu \mathrm{M}$ final concentration), an ATP synthase inhibitor, was injected followed by exposure of carbonylcyanide p-trifluoromethoxyphenylhydrazone (FCCP) (1 $\mu \mathrm{M}$ final concentration), an ETC accelerator which causes maximal respiration and finally rotenone plus antimycin $\mathrm{A}$ (1 $\mu \mathrm{M}$ final concentration of each) which are mitochondrial complex I and III inhibitors into the cell plate, respectively. EC-1 and Eca-109 cells treated with or without paclitaxel included three replicates and the results were obtained by performing three independent experiments.

Statistical analysis. All statistical analyses were carried out using SPSS version 16.0 statistical software package (SPSS Inc., Chicago, IL, USA) and presented with GraphPad Prism version 5.0 software (GraphPad Software, Inc., La Jolla, CA, USA). All P-values are two-sided, and $\mathrm{p}<0.05$ was considered a statistically significant difference. Error bars for the experiments represent the standard deviation of the mean value (mean $\pm \mathrm{SD}$ ) from three separate experiments.

\section{Results}

Paclitaxel inhibits EC-1 and Eca-109 cell growth and increases intracellular ROS in a time-dependent manner. The anti-proliferative effect of paclitaxel on EC-1 and Eca-109 cells was determined by performing an MTT assay. We found that paclitaxel treatment reduced the ESCC cell viability in a time-dependent manner (Fig. 1A). In addition, we determined the intracellular ROS production induced by 

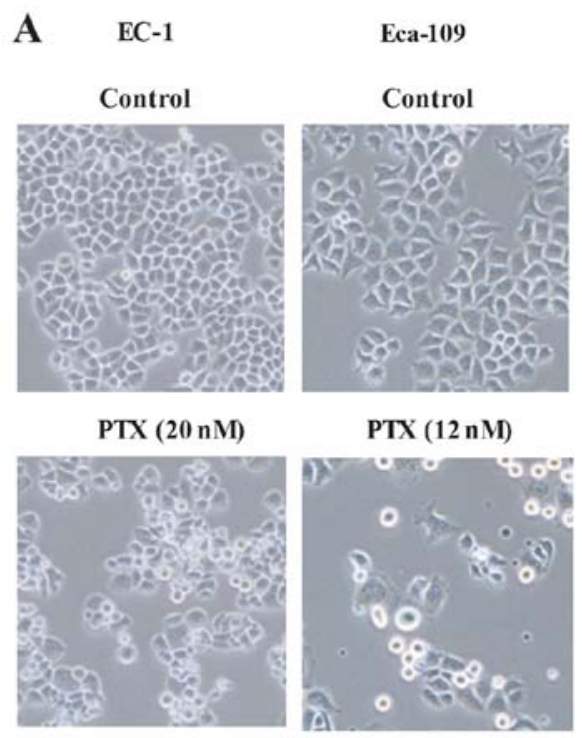

C

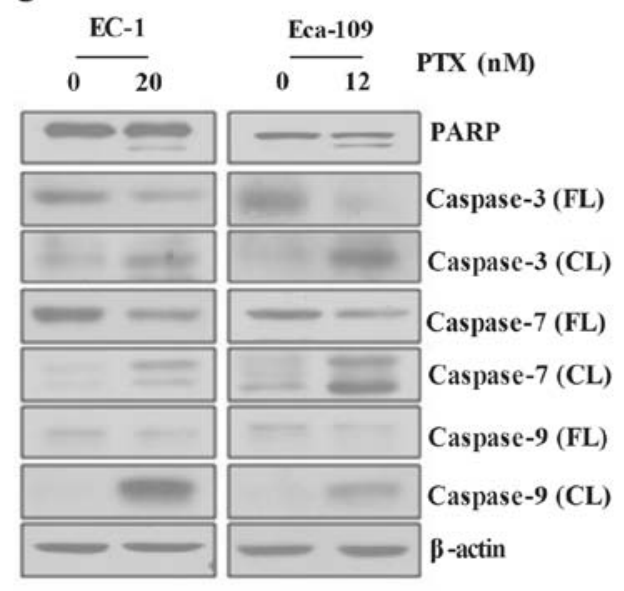

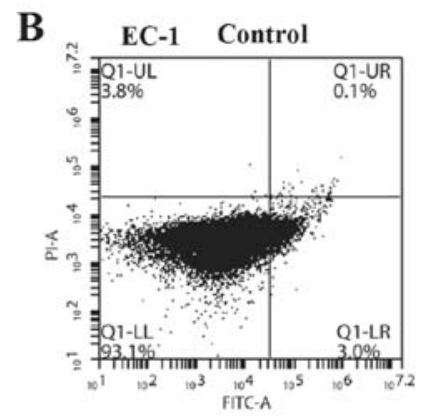
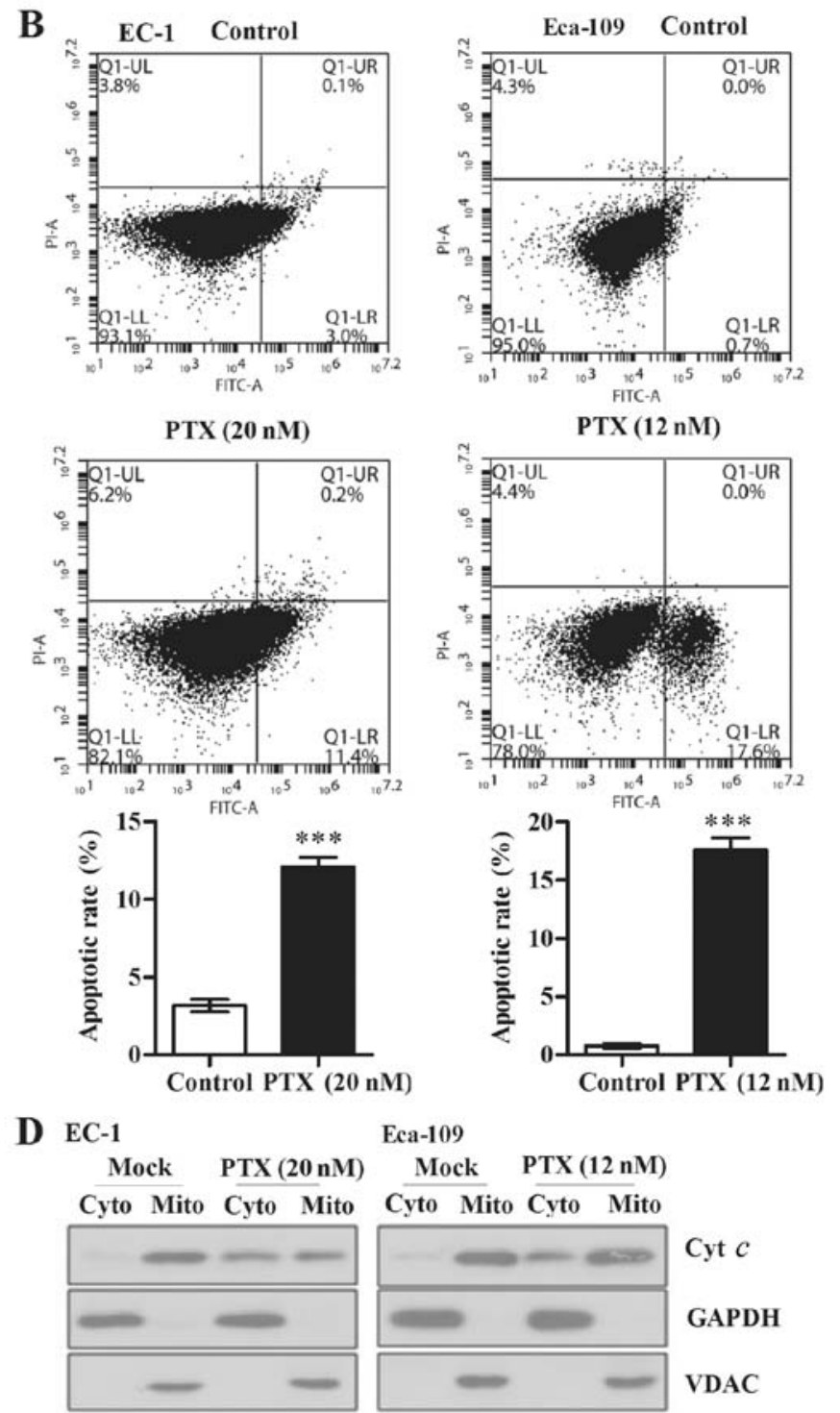

Eca-109

Mock PTX (12 nM) Cyto Mito Cyto Mito

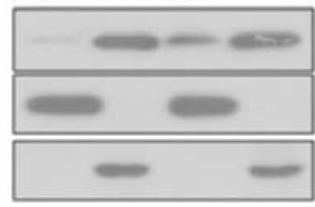

Cyt $c$

GAPDH

VDAC

Figure 2. Paclitaxel induces apoptosis in EC-1 and Eca-109 cells. (A) EC-1 and Eca-109 cells treated with paclitaxel resulted in cell apoptosis as shown by optical microscopy. (B) Flow cytometry results of EC-1 and Eca-109 cells treated with 20 and 12 nM paclitaxel, respectively. (C) Paclitaxel (20 and 12 nM) treatment increased the cleavage of PARP, caspase-3, caspase-7 and caspase-9. (D) The representative western blot analysis showed that paclitaxel (20 and 12 nM) treatment to EC-1 and Eca-109 cells stimulated the release of cytochrome $c$ into cytoplasm. ${ }^{* * *} \mathrm{p}<0.001$.

paclitaxel treatment, and our results showed that paclitaxel treatment significantly promoted ROS generation in EC-1 and Eca-109 cells (Fig. 1B).

Paclitaxel induces apoptosis in EC-1 and Eca-109 cells. We next examined the effect of paclitaxel on apoptosis of ESCC cells. Optical microscopy revealed a significant increase in cell apoptosis (Fig. 2A) and the flow cyto-metry results showed that apoptotic rates were increased from 2.6 and $0.3 \%$ (DMSO control) to $23.5 \%$ ( $\mathrm{p}<0.01)$ and $16.4 \%$ ( $\mathrm{p}<0.001)$ when treated with 12 and $20 \mathrm{nM}$ paclitaxel for $24 \mathrm{~h}$ in Eca-109 and EC-1 cells, respectively (Fig. 2B). Additionally, we found that cleaved PARP, cleaved caspase-3, cleaved caspase-7 and cleaved caspase- 9 were all markedly increased when Eca-109 and EC-1 cells were treated with paclitaxel (Fig. 2C). Moreover, our results showed that paclitaxel treatment led to increased release of cytochrome $c$ from mitochondria to cytoplasm (Fig. 2D).
Paclitaxel reduces MMP and STAT3 phosphorylation at Ser727 in EC-1 and Eca-109 cells. JC-1 is a membrane-permeable lipophilic dye that exists as J-aggregates in the mitochondrial matrix (red fluorescence) and as monomers in the cytoplasm (green fluorescence). During mitochondrial depolarization, the red J-aggregates form green monomers due to a change in MMP. Thus, depolarization can be measured as an increasing green fluorescent/red fluorescent intensity ratio. Our results showed that paclitaxel causes a significant loss of MMP in EC-1 and Eca-109 cells (Fig. 3A; p<0.1). In addition, we analyzed the protein levels of total and phosphorylated (Ser727) STAT3 in EC-1 and Eca-109 cells by western blot analysis, and our results showed that paclitaxel treatment led to significantly decreased total and phosphorylated (Ser727) STAT3 protein levels (Fig. 3B and C; p<0.05). Moreover, the knockdown of STAT3 in Eca-109 and EC-1 cells enhanced paclitaxel induced PARP and caspase- 3 cleavage in Eca-109 and EC-1 cells (Fig. 3D). 
A
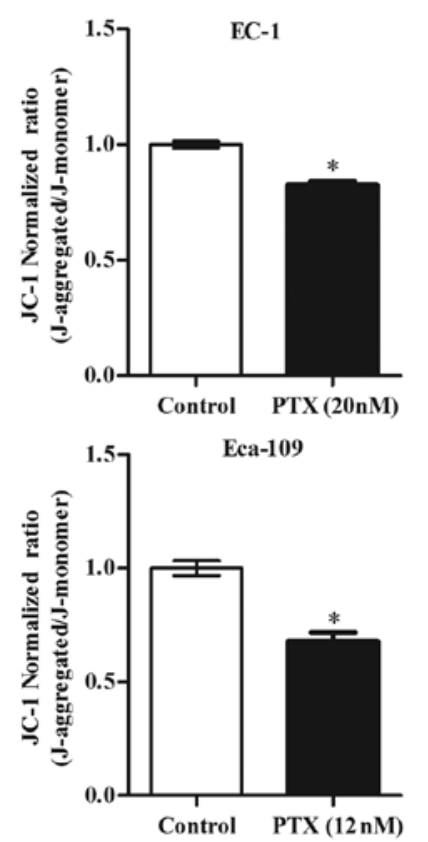

B

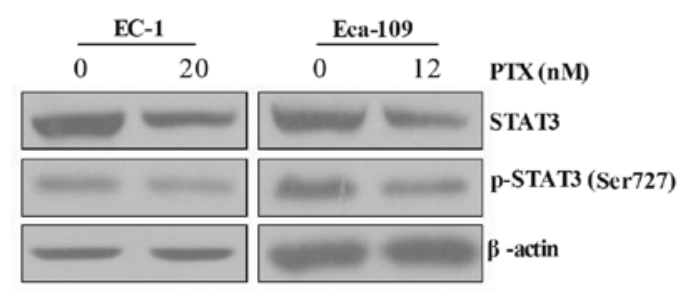

C

EC-1

Eca-109
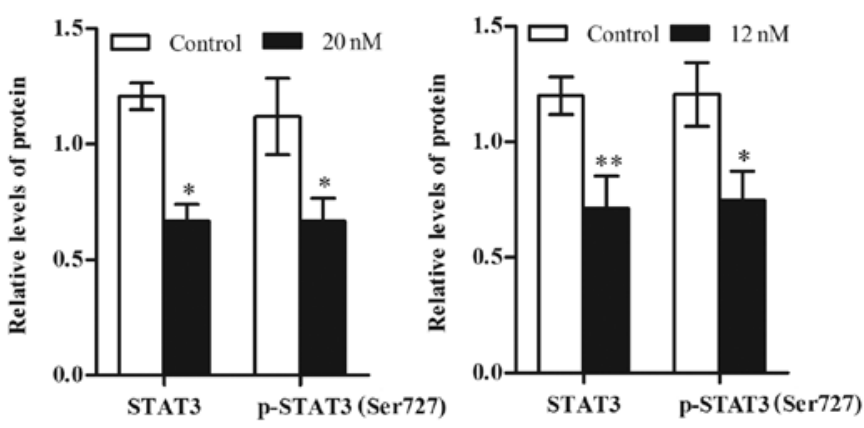

D

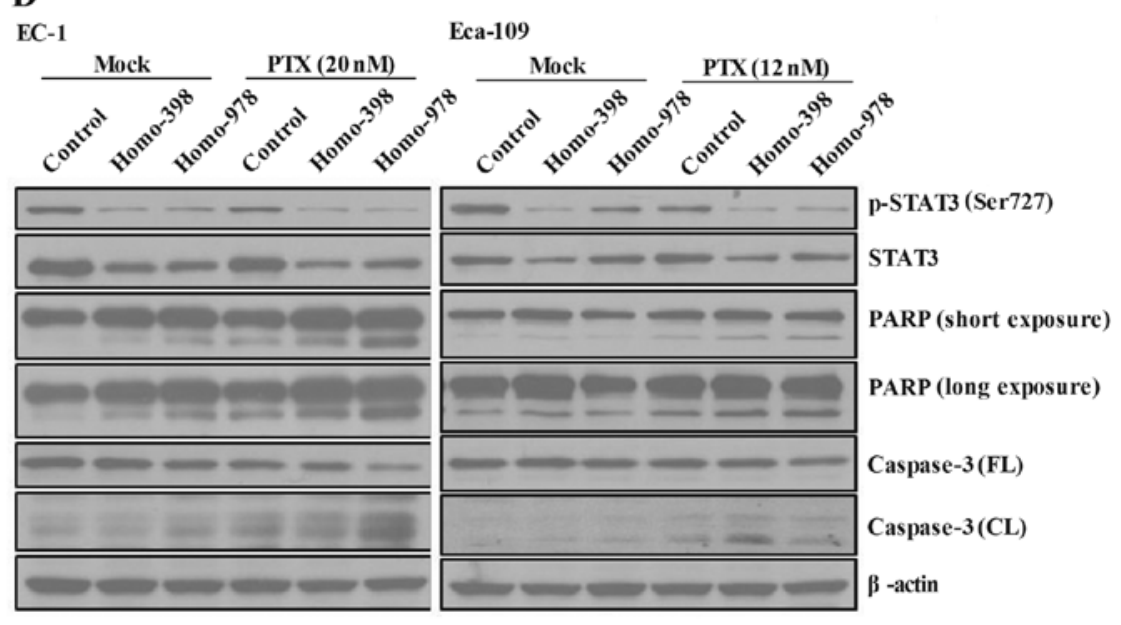

Figure 3. Paclitaxel reduces MMP and STAT3 phosphorylation at Ser727 in EC-1 and Eca-109 cells. (A) EC-1 and Eca-109 cells were treated with paclitaxel (12 and $20 \mathrm{nM}$ ), and MMP were measured by staining the cells with JC-1 and analyzed by flow cytometry. The ratio of fluorescence intensities Ex/Em=490/590 and 490/530 nm (FL590/FL530) were recorded to show the MMP level of each sample. Data are presented as mean \pm SD ( $n=3$ ). (B) Representative western blot analysis showed that paclitaxel (12 and $20 \mathrm{nM}$ ) treatment led to cellular STAT3 and phospho-STAT3 (Ser727) downregulation. (C) The relative protein level of cellular STAT3 and phospho-STAT3 (Ser727) were quantified using ImageJ software. (D) Representative western blot analysis showed that STAT3knockdown enhanced paclitaxel induced PARP and caspase-3 cleavage in Eca-109 and EC-1 cells. MMP, mitochondrial membrane potential; STAT3, signal transducer and activator of transcription $3 .{ }^{*} \mathrm{p}<0.05 ;{ }^{* *} \mathrm{p}<0.01$.

Paclitaxel mainly decreases mtSTAT3 and phosphorylated (Ser727) STAT3 in EC-1 and Eca-109 cells. STAT3 is also detected in the mitochondria and has functions in regulating glycolysis and OXPHOS of cancer cells. To explore which part of total and phosphorylated (Ser727) STAT3 of EC-1 and Eca-109 cells was reduced after paclitaxel treatment, mitochondrial and cytoplasmic fraction were isolated, and the proteins levels of both total and phosphorylated (Ser727) STAT3 were detected by western blot analysis. Our results showed that paclitaxel treatment reduced total and phosphorylated (Ser727) STAT3 in the mitochondria, but did not decrease the total and phosphorylated (Ser727) STAT3 in the cytosol. GAPDH was used as a cytoplasmic loading control and VDAC was used as a mitochondrial loading control (Fig. 4A). To further clarify the extent of reduction of total and phosphorylated (Ser727) STAT3, we used loading control to normalize total and phosphorylated (Ser727) STAT3 protein level, and the results are statistically significant (Fig. 4B; p<0.05).

Paclitaxel inhibits mitochondrial respiration in EC-1 and Eca-109 cells. Next, we detected the OCR in EC-1 and Eca-109 cells by Seahorse XF96 extracellular flux analyzer. Paclitaxel treatment decreased OCR of EC-1 and Eca-109 cells markedly (Fig. 5A-C). Furthermore, the ATP production was strongly reduced in response to paclitaxel treatment (Fig. 5D), which indicates that paclitaxel treatment may impair the OXPHOS of cancer cells. 
$\mathbf{A}$

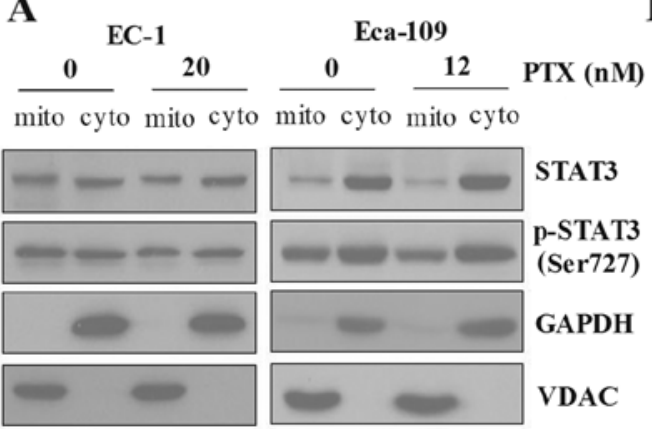

B

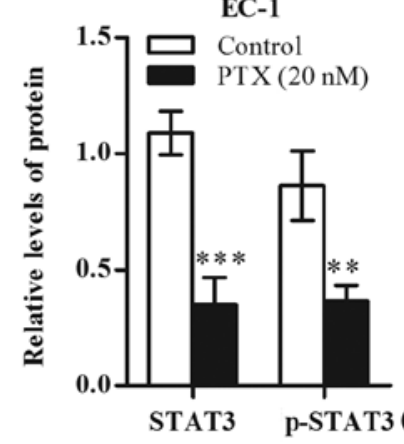

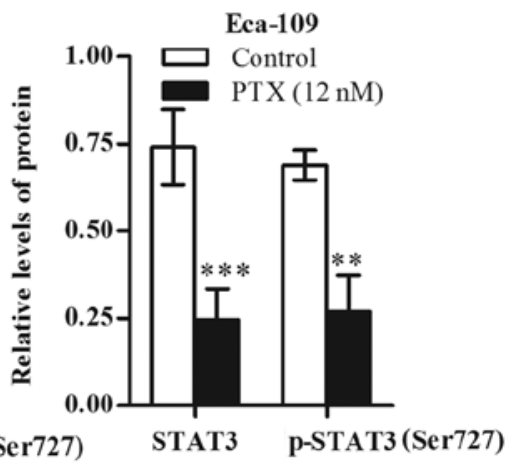

Figure 4. Paclitaxel mainly decreases mitochondrial STAT3 phosphorylation at Ser727 of EC-1 and Eca-109 cells. (A) Representative western blot analysis indicated that EC-1 and Eca-109 cells treated with paclitaxel decreased both mtSTAT3 and phosphor-mtSTAT3 (Ser727) levels. (B) The mtSTAT3 and phospho-mtSTAT3 (Ser727) levels were quantified using ImageJ software. STAT3, signal transducer and activator of transcription $3 .{ }^{* *} \mathrm{p}<0.01,{ }^{* * * *} \mathrm{p}<0.001$.

A

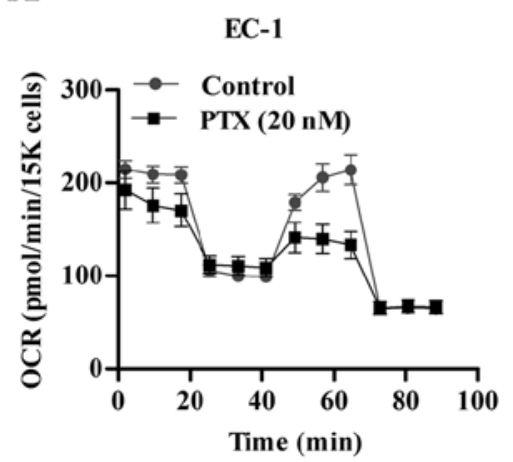

Eca-109

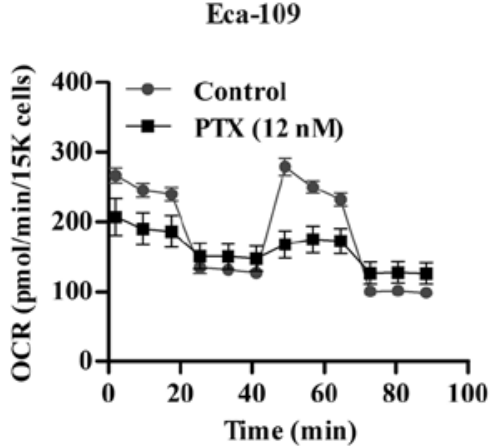

B

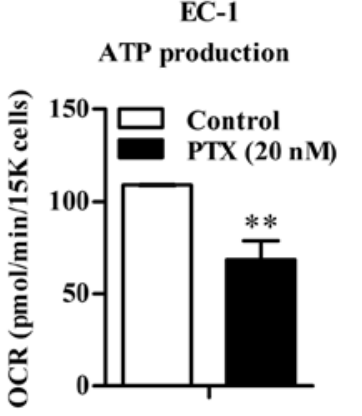

Eca-109

ATP production

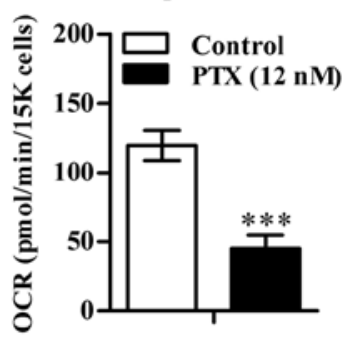

C

C

Basal respiration

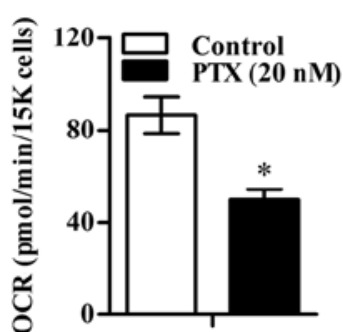

Eca-109 Basal respiration

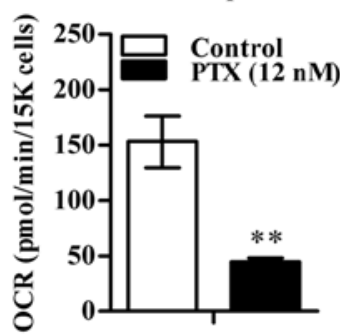

D

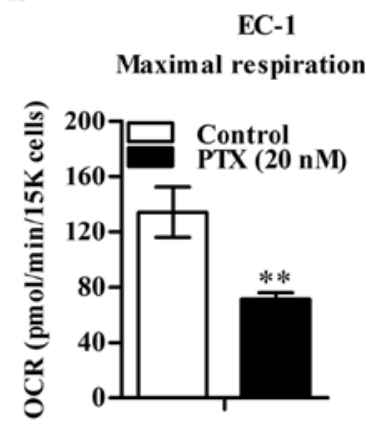

Figure 5. Paclitaxel inhibits mitochondrial respiration in EC-1 and Eca-109 cells. (A) Mitochondrial respiration profile of paclitaxel-treated EC-1 and Eca-109 cells. The intact cellular OCR was measured in real-time using the Seahorse XF96 extracellular flux analyzer. Basal OCR was measured at three time-points, followed by sequential injection of the ATP synthase inhibitor oligomycin $(1 \mu \mathrm{M})$, the uncoupler FCCP $(1 \mu \mathrm{M})$, the complex I inhibitor rotenone $(1 \mu \mathrm{M})$ and complex III inhibitor antimycin A $(1 \mu \mathrm{M})$. The representative graph represents the mean OCR \pm SD of six replicates. (B) ATP production of control (DMSO) and paclitaxel-treated EC-1 and Eca-109 cells were calculated by the OCR of baseline minus oligomycin treatment. (C and D) Basal and maximal respiration (OCR) of control (DMSO) and paclitaxel treated EC-1 and Eca-109 cells. Data are presented as mean $\pm \mathrm{SD}\left(\mathrm{n}=6,{ }^{*} \mathrm{p}<0.05,{ }^{* *} \mathrm{p}<0.01\right.$, *** $\mathrm{p}<0.001)$. OCR, oxygen consumption rate.

\section{Discussion}

Paclitaxel is widely used as a common clinical medicine in the treatment of solid tumors $(23,24)$. In this study, we demonstrated that paclitaxel inhibits the cell viability significantly in both Eca-109 and EC-1 cells through inducing apoptosis of ESCC cells by downregulation of STAT3 Ser727 phosphorylation.

Understanding the mechanism of ROS generation may provide a new method for the development of therapeutic agents that are capable of selectively inducing apoptosis of cancer cells. ROS generation has been shown to be a common cellular mechanism for multiple cell death pathways, including gene activation, cell cycle arrest and apoptosis (21). ROS may also serve as a signal for apoptosis instead of being the consequence of the cellular changes induced by apoptosis (22). ROS generation may alter the redox-state of cells and the sensitivity of cells to apoptotic stimulus and ultimately trigger the subsequent apoptotic events (25-28). Our results showed that paclitaxel treatment resulted in the disruption of MMP, 
increased ROS generation, and thus promoted apoptosis in Eca-109 and EC-1 cells.

It has been demonstrated that STAT3 also located in mitochondria (18). In the present study, we found that paclitaxel treatment significantly decreased the total and Ser727 phosphorylated STAT3 level in mitochondria of Eca-109 and EC-1 cells. These results suggest that paclitaxel treatment could reduce the phosphorylation level of STAT3. The reason why total STAT3 changed in paclitaxel-induced ESCC cells is probably associated with the feedback of ESCC cells to paclitaxel treatment as an integrated system.

STAT3 was able to positively regulate the mitochondrial respiration in terminally differentiated cells $(29,30)$ and mtSTAT3 increased the activity of complex I and II in the ETC in a transcriptional-independent manner (18). STAT3 was also linked to carcinogenesis and tumor development shown in previous studies (31-33). Our results indicated that the OCR significantly decreased in paclitaxel-treated Eca-109 and EC-1 cells, which may be attributed to the decrease of mitochondrial respiratory chain enzyme activities. In addition, mitochondrial cytochrome $c$, which is a downstream regulation factor of STAT3, also significantly increased in the cytosol of both Eca-109 and EC-1 cells, which indicates the loss of MMP caused the leakage of cytochrome $\mathrm{c}$ due to the paclitaxel treatment of ESCC cells. It is well known that the increment of ROS level and the deceasing of MMP may induce apoptosis through caspase- 3 activation and cytochrome $c$ release $(28,34,35)$, the increasing cytochrome $c$ protein level in the cytosol of both Eca-109 and EC-1 cells suggested the paclitaxel-induced mitochondria-dependent apoptotic pathway. Moreover, depletion of STAT3 increased the sensitivity of ESCC cells to paclitaxel through enhancing the cleavage of PARP and caspase-3.

In conclusion, our findings demonstrated that paclitaxel has significant anti-proliferation effects by inducing mitochondrial apoptosis of ESCC cells via STAT3 signaling pathways. Thus, our data provide a novel insight into the mitochondrial apoptosis mechanism of paclitaxel on the ESCC cells in vitro. These findings suggested that paclitaxel may be of therapeutic potential in ESCC treatment through the induction of mitochondrial apoptosis in ESCC cells.

\section{Acknowledgements}

The authors would like to thank Dr Sushil Kumar for the critical review of the manuscript. This study was supported by the grant from the National Natural Science Foundation of China (nos. 31171345 and 31570772 ) to B.L. and the Natural Science Foundation of Zhejiang Province (LY17C070005) to Y.L.

\section{References}

1. Kamangar F, Dores GM and Anderson WF: Patterns of cancer incidence, mortality, and prevalence across five continents: defining priorities to reduce cancer disparities in different geographic regions of the world. J Clin Oncol 24: 2137-2150, 2006.

2. Tang WR, Fang JY, Wu KS, Shi XJ, Luo JY and Lin K: Epidemiological characteristics and prediction of esophageal cancer mortality in China from 1991 to 2012. Asian Pac J Cancer Prev 15: 6929-6934, 2014.
3. Song QK, Li J, Jiang HD, He YM, Zhou XQ and Huang CY: Esophageal cancer mortality during 2004-2009 in Yanting County, China. Asian Pac J Cancer Prev 13: 5003-5006, 2012.

4. Xu Y, Yu X, Chen Q and Mao W: Neoadjuvant versus adjuvant treatment: which one is better for resectable esophageal squamous cell carcinoma? World J Surg Oncol 10: 173, 2012.

5. Blot WJ: Esophageal cancer trends and risk factors. Semin Oncol 21: 403-410, 1994.

6. Brooks-Brunn JA: Esophageal cancer: an overview. Medsurg Nurs 9: 248-254, 2000.

7. Scripture CD, Figg WD and Sparreboom A: Paclitaxel chemotherapy: from empiricism to a mechanism-based formulation strategy. Ther Clin Risk Manag 1: 107-114, 2005.

8. Ajani JA, Ilson DH, Daugherty K, Pazdur R, Lynch PM and Kelsen DP: Activity of taxol in patients with squamous cell carcinoma and adenocarcinoma of the esophagus. J Natl Cancer Inst 86: 1086-1091, 1994.

9. Ilson DH, Ajani J, Bhalla K, Forastiere A, Huang Y, Patel P, Martin L, Donegan J, Pazdur R, Reed C, et al: Phase II trial of paclitaxel, fluorouracil, and cisplatin in patients with advanced carcinoma of the esophagus. J Clin Oncol 16: 1826-1834, 1998.

10. Adelstein DJ, Rice TW, Rybicki LA, Larto MA, Ciezki J, Saxton J, DeCamp M, Vargo JJ, Dumot JA and Zuccaro G: Does paclitaxel improve the chemoradiotherapy of locoregionally advanced esophageal cancer? A nonrandomized comparison with fluorouracil-based therapy. J Clin Oncol 18: 2032-2039, 2000.

11. Polee MB, Eskens FA, van der Burg ME, Splinter TA, Siersema PD, Tilanus HW, Verweij J, Stoter G and van der Gaast A: Phase II study of bi-weekly administration of paclitaxel and cisplatin in patients with advanced oesophageal cancer. Br J Cancer 86: 669-673, 2002.

12. Tishler RB, Schiff PB, Geard CR and Hall EJ: Taxol: a novel radiation sensitizer. Int J Radiat Oncol Biol Phys 22: 613-617, 1992.

13. Choy H, Rodriguez FF, Koester S, Hilsenbeck S and Von Hoff DD: Investigation of taxol as a potential radiation sensitizer. Cancer 71: 3774-3778, 1993.

14. Leonard CE, Chan DC, Chou TC, Kumar R and Bunn PA: Paclitaxel enhances in vitro radiosensitivity of squamous carcinoma cell lines of the head and neck. Cancer Res 56: 5198-5204, 1996.

15. Catlett-Falcone R, Landowski TH, Oshiro MM, Turkson J, Levitzki A, Savino R,Ciliberto G, Moscinski L,Fernández-Luna JL, Nuñez G, et al: constitutive activation of Stat 3 signaling confers resistance to apoptosis in human U266 myeloma cells. Immunity 10: 105-115, 1999.

16. Fukada T, Hibi M, Yamanaka Y, Takahashi-Tezuka M, Fujitani Y, Yamaguchi T, Nakajima K and Hirano T: Two signals are necessary for cell proliferation induced by a cytokine receptor gp130: involvement of STAT3 in anti-apoptosis. Immunity 5: 449-460, 1996.

17. Yan S, Zhou C, Zhang W, Zhang G, Zhao X, Yang S, Wang Y, Lu N, Zhu H and Xu N: Beta-catenin/TCF pathway upregulates STAT3 expression in human esophageal squamous cell carcinoma. Cancer Lett 271: 85-97, 2008.

18. Wegrzyn J, Potla R, Chwae YJ, Sepuri NB, Zhang Q, Koeck T, Derecka M, Szczepanek K, Szelag M, Gornicka A, et al: Function of mitochondrial Stat 3 in cellular respiration. Science 323: 793-797, 2009.

19. Gough DJ, Corlett A, Schlessinger K, Wegrzyn J, Larner AC and Levy DE: Mitochondrial STAT3 supports Ras-dependent oncogenic transformation. Science 324: 1713-1716, 2009.

20. Blajeski AL, Kottke TJ and Kaufmann SH: A multistep model for paclitaxel-induced apoptosis in human breast cancer cell lines. Exp Cell Res 270: 277-288, 2001.

21. Ofir R, Seidman R, Rabinski T, Krup M, Yavelsky V, Weinstein Y and Wolfson M: Taxol-induced apoptosis in human SKOV3 ovarian and MCF7 breast carcinoma cells is caspase-3 and caspase-9 independent. Cell Death Differ 9: 636-642, 2002.

22. Varbiro G, Veres B, Gallyas F Jr and Sumegi B: Direct effect of Taxol on free radical formation and mitochondrial permeability transition. Free Radic Biol Med 31: 548-558, 2001.

23. Liu X, Kim CN, Yang J, Jemmerson R and Wang X: Induction of apoptotic program in cell-free extracts: requirement for dATP and cytochrome $c$. Cell 86: 147-157, 1996.

24. Susin SA, Lorenzo HK, Zamzami N, Marzo I, Snow BE, Brothers GM, Mangion J, Jacotot E, Costantini P, Loeffler M, et al: Molecular characterization of mitochondrial apoptosis-inducing factor. Nature 397: 441-446, 1999. 
25. Johnson TM, Yu ZX, Ferrans VJ, Lowenstein RA and Finkel T: Reactive oxygen species are downstream mediators of p53-dependent apoptosis. Proc Natl Acad Sci USA 93: 11848-11852, 1996.

26. Cai J and Jones DP: Mitochondrial redox signaling during apoptosis. J Bioenerg Biomembr 31: 327-334, 1999.

27. Simon HU, Haj-Yehia A and Levi-Schaffer F: Role of reactive oxygen species (ROS) in apoptosis induction. Apoptosis 5: 415-418, 2000.

28. Akgul C, Moulding DA and Edwards SW: Molecular control of neutrophil apoptosis. FEBS Lett 487: 318-322, 2001.

29. Zouein FA, Duhé RJ, Arany I, Shirey K, Hosler JP, Liu H, Saad I, Kurdi M and Booz GW: Loss of STAT3 in mouse embryonic fibroblasts reveals its janus-like actions on mitochondrial function and cell viability. Cytokine 66: 7-16, 2014.

30. Carbognin E, Betto RM, Soriano ME, Smith AG and Martello G: Stat3 promotes mitochondrial transcription and oxidative respiration during maintenance and induction of naive pluripotency. EMBO J 35: 618-634, 2016.
31. Boengler K, Hilfiker-Kleiner D, Heusch G and Schulz R: Inhibition of permeability transition pore opening by mitochondrial STAT3 and its role in myocardial ischemia/reperfusion. Basic Res Cardiol 105: 771-785, 2010.

32. Nguyen AV, Wu YY, Liu Q, Wang D, Nguyen S, Loh R, Pang J, Friedman K, Orlofsky A, Augenlicht L, et al: STAT3 in epithelial cells regulates inflammation and tumor progression to malignant state in colon. Neoplasia 15: 998-1008, 2013.

33. Haricharan S and Li Y: STAT signaling in mammary gland differentiation, cell survival and tumorigenesis. Mol Cell Endocrinol 382: 560-569, 2014.

34. Ly JD, Grubb DR and Lawen A: The mitochondrial membrane potential (deltapsi(m)) in apoptosis; an update. Apoptosis 8: 115-128, 2003

35. Wang $C$ and Youle RJ: The role of mitochondria in apoptosis. Annu Rev Genet 43: 95-118, 2009. 